\section{Endoscopic ultrasound-guided esophago- enterostomy for a completely obstructed surgical anastomosis}

Endoscopic ultrasound (EUS) has the potential to safely guide the creation of gastrointestinal anastomoses [1]. Different methods have been studied in animal models [2] and more recently EUS-guided gastroenterostomy with a lumen-apposing stent has been described in two small patient series $[3,4]$.

A 58-year-old woman with junctional adenocarcinoma who had undergone total gastrectomy with distal esophagectomy in October 2014 was referred to us with grade 4 dysphagia in January 2015. Endoscopy revealed a completely obstructed esophagojejunal anastomosis, the position of which was confirmed by imaging (৫ Fig. 1).

Initial access to the jejunum was accomplished under EUS guidance with a flexible 19G needle (Expect; Boston Scientific, Spencer, Indiana, USA) ( Fig.2). Once the tip of the needle had been advanced beyond the anastomosis, contrast agent was injected to confirm that the needle was correctly located in the jejunum. A 0.025-inch guidewire was advanced through the needle and looped in the intestinal lumen. An over-the-wire exchange with a therapeutic forwardviewing endoscope was performed and the anastomotic tract was dilated over the guidewire, first with a 7-Fr biliary dilation catheter, then up to $6 \mathrm{~mm}$ using a biliary balloon. Finally, a fully-covered biliary self-expanding metal stent (SEMS) was inserted and left in place for 1 week to prevent anastomotic leakage and to consolidate the passage ( $\mathbf{F i g} \cdot \mathbf{3 a}$ ).

The biliary stent was subsequently replaced with a fully-covered "yo-yo" stent (NAGI stent; Taewoong, Seoul, Korea) with a $16-\mathrm{mm}$ diameter ( $\bullet$ Fig. 3 b). However, because of pain, this stent was removed after only 1 week and over three consecutive sessions the neoanastomosis was progressively dilated with balloons up to $20 \mathrm{~mm}$ ( $\bullet \mathrm{Fig.3c}$; $\bullet$ Video 1 ).

This is the first reported case of EUS-guided treatment of a completely obstructed esophagoenteric anastomosis located in the mediastinum. EUS helped to achieve safe access to the jejunum by avoiding inadvertent puncture of the surrounding
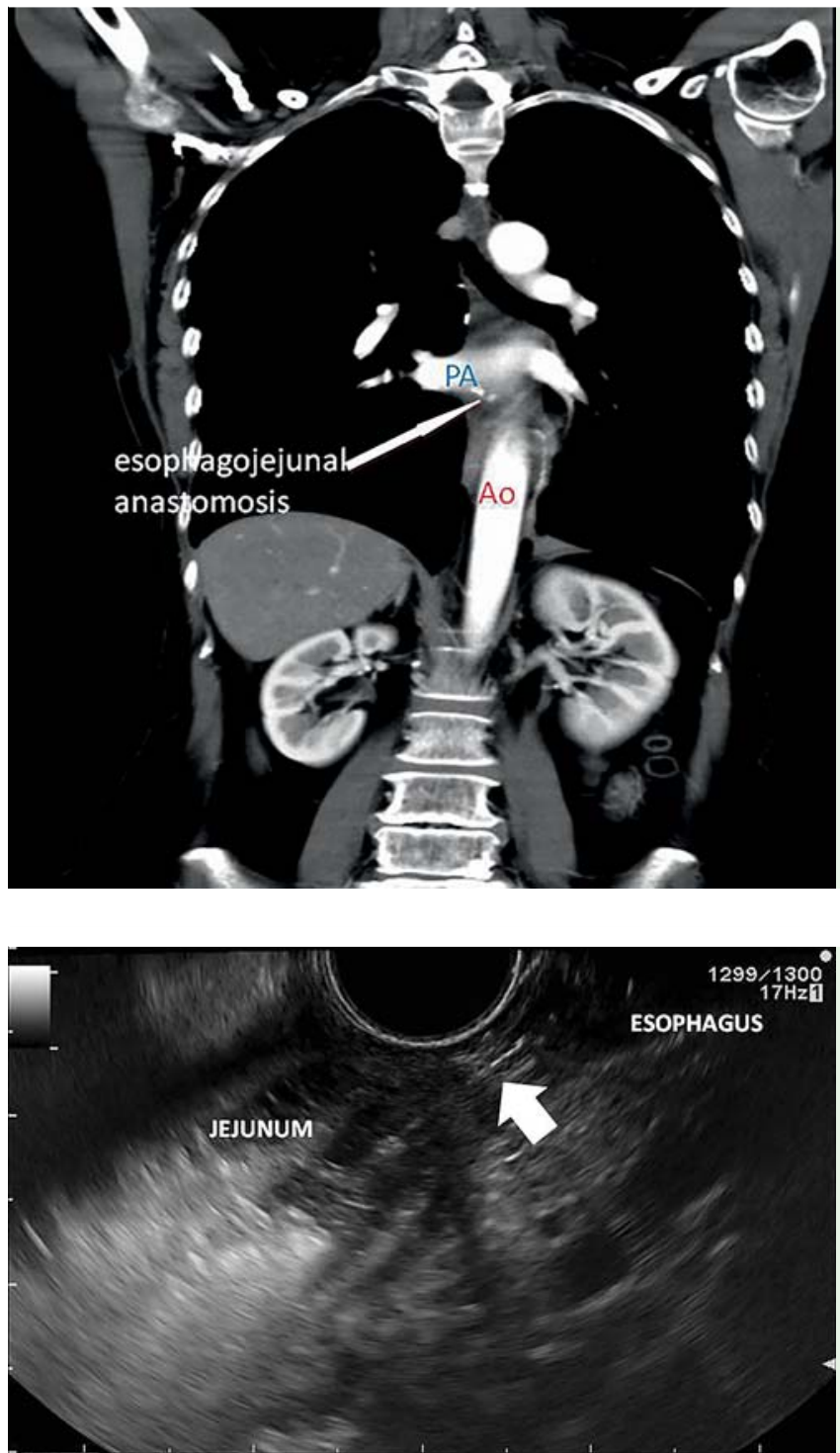

Fig. 1 Imaging performed following the initial endoscopy showing the location of the completely obstructed esophagojejunal anastomosis (white arrow) beyond the pulmonary artery (PA) and in front of the descending aorta (Ao).

Fig. 2 View during endoscopic ultrasound (EUS)-guided placement of a 19G flexible needle (white arrow), which was passed through the esophagus into the jejunum.

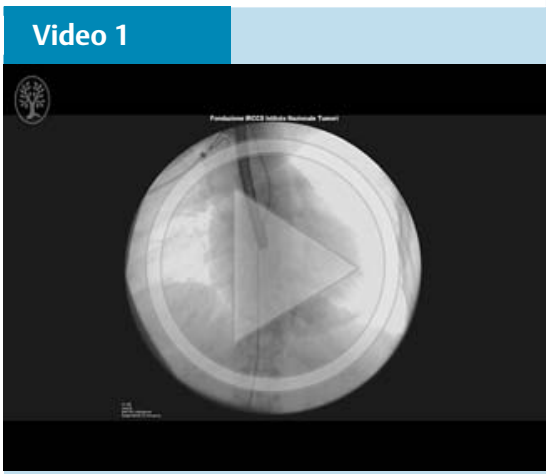

The creation under endoscopic ultrasound (EUS) guidance of an esophagoenterostomy for a completely obstructed esophagojejunal anastomosis. Initial imaging shows the location of the obstructed esophagojejunal anastomosis (PA, pulmonary artery; Ao, descending aorta). A $19 \mathrm{G}$ needle is used to access the jejunum under EUS guidance; a guidewire is passed; an over-the-wire exchange with a therapeutic endoscope is performed; the anastomotic tract is dilated with a biliary catheter then a balloon; a fully covered self-expanding metal stent (SEMS) is placed. After 1 week an ultrathin endoscope is passed through the neoanastomosis, before being exchanged for a therapeutic endoscope; a fully covered "yoyo" metal stent is placed. Finally, on the third endoscopy session following removal of the stent, the neoanastomosis is shown being dilated by a balloon up to $20 \mathrm{~mm}$. 


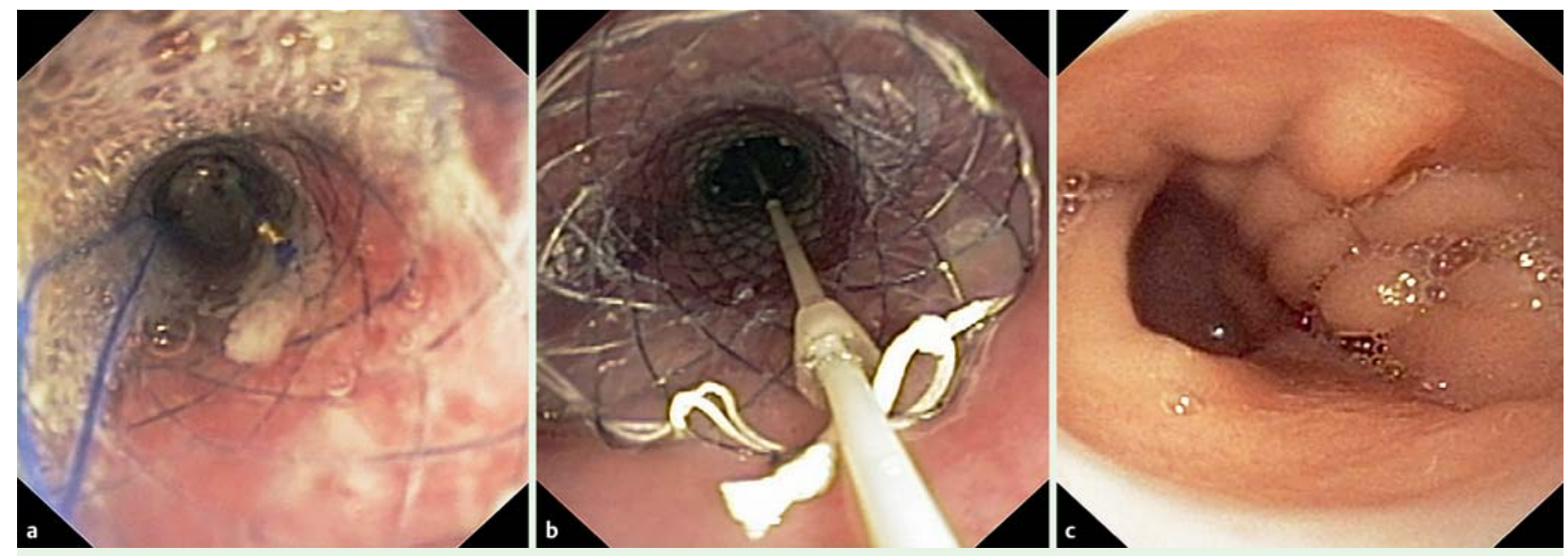

Fig. 3 Endoscopic views showing: a the fully covered biliary self-expanding metal stent (SEMS) that was initially left in place to consolidate the passage and prevent anastomotic leakage; $\mathbf{b}$ the fully covered lumen-apposing stent that was placed instead of the biliary SEMS to dilate the neoanastomosis; $\mathbf{c}$ the appearance of the neoanastomosis after three sessions of balloon dilation.

mediastinal structures, such as the pulmonary artery, descending aorta, and inferior vena cava, which were located close to the anastomosis. No immediate or delayed major complications were observed and patient was able to return to a regular diet on completion of the endoscopic treatment.

Endoscopy_UCTN_Code_TTT_1AS_2AB

\section{Competing interests: None}

\section{Gabriele Delconte ${ }^{1,2}$, Andrea Mancini ${ }^{1}$, Frank P. Vleggaar ${ }^{3}$, Monica Salvetti ${ }^{4}$, Vincenzo Mazzaferro ${ }^{5}$, Enzo Masci $^{1}$}

${ }^{1}$ Diagnostic and Therapeutic Endoscopy Unit, Fondazione IRCCS Istituto Nazionale Tumori, Milan, Italy

2 Department of Oncology (DIPO), University of Milan, Italy
${ }^{3}$ Department of Gastroenterology and Hepatology, University Medical Center Utrecht, Utrecht, The Netherlands

${ }^{4}$ Department of Radiology, Fondazione IRCCS Istituto Nazionale Tumori, Milan, Italy

${ }^{5}$ Department of Surgery, Fondazione IRCCS Istituto Nazionale Tumori, Milan, Italy

\section{References}

1 Khashab MA, Baron TH, Binmoeller KF et al. EUS-guided gastroenterostomy: a new promising technique in evolution. Gastrointest Endosc 2015; 81: $1234-1236$

2 Binmoeller KF, Shah JN. Endoscopic ultrasound-guided gastroenterostomy using novel tools designed for transluminal therapy: a porcine study. Endoscopy 2012; 44: 499-503

3 Khashab MA, Kumbhari V, Grimm IS et al. EUS-guided gastroenterostomy: the first U.S. clinical experience (with video). Gastrointest Endosc 2015; 82: $932-938$
4 Taunk P, Cosgrove N, Loren DE et al. Endoscopic ultrasound-guided gastroenterostomy using a lumen-apposing self-expanding metal stent for decompression of afferent loop obstruction. Endoscopy 2015; 47 (Suppl. 01): E395-E396

\section{Bibliography}

Dol http://dx.doi.org/

10.1055/s-0041-110591

Endoscopy 2016; 48: E1-E2

(c) Georg Thieme Verlag KG

Stuttgart · New York

ISSN 0013-726X

\section{Corresponding author \\ Gabriele Delconte, MD}

Diagnostic and Therapeutic Endoscopy Unit Department of Surgery

Fondazione IRCCS Istituto Nazionale Tumori

Via G. Venezian 1

20133, Milan

Italy

gabriele.delconte@istitutotumori.mi.it 